\title{
Melhorando a atenção dos estudantes através da tutoria de mindfulness em sistemas tutores inteligentes
}

\author{
Antônio A. Alves ${ }^{1}$, Felipe de Morais ${ }^{1}$, Bruno Schaab ${ }^{1}$, Patrícia A. Jaques ${ }^{1}$ \\ ${ }^{1}$ Programa de Pós Graduação em Computação Aplicada (PPGCA) \\ Universidade do Vale do Rio dos Sinos (UNISINOS), São Leopoldo - RS - Brasil \\ \{antonioaugusto, felipmorais,brunoschaab\}@edu.unisinos.br, pjaques@unisinos.br
}

\begin{abstract}
Students who are able to maintain attention present a better performance in school activities. In the school setting, mindfulness has shown to be a relevant technique for the improvement of the students' attention. This work describes the integration of a computational agent into an intelligent tutoring system that is responsible for guiding a mindfulness practice that is adapted to the students' mood and previous experience in meditation. We have conducted an experiment with a duration of 7 weeks, counting with 41 students from the 7 th grade. The results of this experiment, obtained through the application of an attention scale, showed that the practice of mindfulness in a guided and personalized way improves students' attention. However, possibly due to the duration of the experiment, we haven't found statistically significant evidence of a positive impact on students' learning.
\end{abstract}

Resumo. Alunos que conseguem manter a atenção apresentam um melhor desempenho nas atividades escolares. No âmbito escolar, o mindfulness tem se mostrado uma técnica relevante para a melhora da atenção de alunos praticantes. Este trabalho descreve a integração de um agente computacional a um sistema tutor inteligente que é responsável por guiar o aluno a praticar exercícios de mindfulness de forma adaptada ao estado de humor e ao tempo de experiência em meditação do estudante. Foi realizado um experimento com duração de 7 semanas, com 41 alunos do $7^{\circ}$ ano. Os resultados deste experimento, obtidos através da aplicação de uma escala de atenção, evidenciaram que a prática de mindfulness de forma guiada e personalizada melhora a atenção dos alunos. No entanto, possivelmente devido à curta duração do experimento, não foram encontradas evidências estatisticamente significativas de um impacto maior na aprendizagem dos alunos.

\section{Introdução}

A habilidade de se manter atento e concentrado é fundamental para uma boa aprendizagem [Ladewig 2017]. Saber autorregular as próprias reações em momentos de adversidade também impacta na capacidade de concentração e na aprendizagem [Tram 2017]. Desta forma, estar concentrado e focado nas atividades é fundamental para obter bons resultados na vida escolar.

Programas de mindfulness têm sido amplamente utilizados em ambientes escolares para promover o bem-estar físico e mental, além de promover uma melhora nas relações interpessoais com colegas e professores. Programas como o MindUp e 
VII Congresso Brasileiro de Informática na Educação (CBIE 2018)

Anais do XXIX Simpósio Brasileiro de Informática na Educação (SBIE 2018)

o Learn2Breathe têm sido aplicados com sucesso em escolas dos EUA e do Canadá [Bostic 2015]. No Brasil, o trabalho desenvolvido por Waldemar e seus colegas (2016) mostrou que a aplicação de um programa baseado em mindfulness e aprendizagem sócioemocional não só é viável, como também colabora para uma melhora na qualidade de vida, habilidades sociais e na aprendizagem dos estudantes.

Mindfulness é um estado de consciência plena em que o indivíduo direciona sua atenção para o momento presente [Kabat-Zinn 2003]. Meditação envolve um conjunto de técnicas ancestrais utilizadas para melhor a atenção (e, portanto, chegar no estado de mindfulness) e a regulação emocional, difundidas pelos monges budistas e hindus [Kabat-Zinn 2003]. Uma das práticas mais difundidas de mindfulness envolve prestar atenção na própria respiração.

Este trabalho visa integrar técnicas de meditação para o treinamento da atenção dos alunos em um Sistema Tutor Inteligente (STI). Essa integração tem como objetivo melhorar a atenção dos alunos, consequentemente, proporcionando uma melhora na aprendizagem. Para atingir esse objetivo, um agente computacional foi desenvolvido para guiar os alunos na realização das práticas de mindfulness. A forma de guiar os alunos nas práticas de meditação ocorre de forma adaptada ao (i) estado de humor do aluno e ao (ii) tempo de experiência do estudante em meditação. A adaptação é realizada com base no autorrelato do aluno sobre seu estado de humor e com base no tempo de prática dos exercícios guiados. Esse agente computacional foi integrado ao STI PAT2Math, que objetiva auxiliar os alunos na resolução de equações de primeiro grau, fornecendo feedbacks específicos a cada interação do aluno com o sistema [Jaques et al. 2013].

Um experimento foi realizado para verificar se a prática guiada e adaptada de mindfulness melhora a atenção do aluno e, por consequência, a sua aprendizagem. $\mathrm{O}$ experimento teve a duração de sete semanas e contou com a participação de 41 alunos do $7^{\circ}$ ano, que foram distribuídos aleatoriamente em três grupos, onde cada grupo utilizou uma versão diferente do STI PAT2Math: meditação não adaptada, meditação adaptada e história da álgebra. O objetivo do experimento era verificar, através das três versões, se a meditação adaptada tem um efeito maior em relação a meditação não adaptada e em relação ao fato de escutar um áudio sobre a história da álgebra.

O diferencial deste trabalho, em relação ao estado da arte, é que a apresentação das técnicas de meditação ao estudante é feita de forma adaptada, levando em consideração o humor e o tempo de experiência do estudante em meditação. Para isso, o sistema armazena o histórico do aluno referente tanto ao humor, quanto ao tempo de exposição às técnicas de meditação. Estudos recentes mostram que o humor negativo ou sentimentos desagradáveis podem ter impacto na saúde e na capacidade de manter a atenção [Goldsby et al. 2017, Yuzheng et al. 2017]. Assim sendo, a identificação do humor permite que o agente selecione uma meditação, em forma de gravação de áudio, que permita ao estudante regular a sua resposta emocional e melhorar a capacidade de atenção de forma mais efetiva.

\section{Trabalhos Relacionados}

Esta seção relata os trabalhos relacionados a este estudo. O objetivo desta seção é apresentar trabalhos que fazem uso de alguma ferramenta computacional utilizada para propiciar e facilitar a prática de exercícios de meditação. 
VII Congresso Brasileiro de Informática na Educação (CBIE 2018)

Anais do XXIX Simpósio Brasileiro de Informática na Educação (SBIE 2018)

Hudlicka (2013) desenvolveu um treinador virtual de mindfulness, implementado como um agente conversacional animado, cujo objetivo era apresentar instruções para a realização de exercícios de meditação. O praticante e o agente interagiam entre si através de diálogo em linguagem natural (escrito). Os resultados obtidos com o estudo piloto indicam que o treinamento realizado com o treinador virtual orientando a meditação é mais eficaz do que a simples apresentação de material instrucional e da prática autorregulada.

O trabalho de Gromala et al. (2015) propõe um ambiente de realidade virtual para a prática de mindfulness como tratamento alternativo para pacientes com dores crônicas. No Virtual Meditative Walk (VMW), o usuário pode caminhar em uma agradável floresta em um ambiente de realidade virtual com o auxílio de uma câmera. $\mathrm{O}$ experimento contou com a participação de 13 indivíduos adultos que foram distribuídos aleatoriamente em grupo de controle e experimental. Os participantes do grupo de controle ouviram uma faixa de áudio com uma técnica da terapia de Mindfulness Based Stress Reduction (MBSR), enquanto os participantes do grupo experimental ouviam a mesma faixa de áudio enquanto utilizavam o equipamento de realidade virtual. Os resultados indicaram que houve uma maior redução no nível de dor relatado pelos indivíduos do grupo experimental do que nos indivíduos do grupo de controle.

D’Mello (2016) propôs uma interface inteligente que detecta a distração da mente através do movimento dos olhos durante a leitura, usando um dispositivo de eye-tracking. Quando a distração mental é detectada, pelo movimento ocular, o sistema intervém (com uma pergunta relacionada ao conteúdo da página) para que o leitor recupere a atenção. Resultados de um estudo experimental com 104 participantes, distribuídos em grupo de controle e experimental, evidenciou que o mau desempenho, ao responder às questões referentes ao conteúdo, estava diretamente relacionado aos episódios de distração durante a leitura, fornecendo assim evidências da validade do detector de distração. Além disso, o grupo experimental teve uma redução significativa nos episódios de distração em relação ao grupo de controle.

O trabalho proposto por Schaab e seus colegas (2015) apresenta um estudo piloto sobre a utilização de áudios para guiar os alunos em uma sessão de mindfulness durante a utilização do STI PAT2Math. O experimento, com duração de quatro sessões, foi realizado com 59 estudantes divididos aleatoriamente em grupo experimental e de controle. Enquanto o grupo de mindfulness utilizava uma gravação para orientar a prática de mindfulness dos alunos, o grupo de controle ouvia uma gravação sobre a história da álgebra. A influência do mindfulness na melhora da aprendizagem foi avaliada através de pré e pós-teste. Não foram encontradas evidências estatisticamente significativas nesse estudo de que o grupo experimental aprendeu mais ou teve uma melhora maior da atenção do que o grupo de controle.

Diferentemente dos trabalhos relacionados citados nesta seção, no trabalho proposto a apresentação das técnicas de meditação ao estudante será feita de forma adaptada, levando em consideração o humor do estudante e seu tempo de experiência em técnicas de meditação. O sistema armazena o histórico do aluno tanto de humor, quanto seu tempo de exposição às técnicas de meditação, para que seja fornecido um treinamento de mindfulness guiado e adaptado para cada estudante. Nenhum dos trabalhos citados busca fornecer instrução de meditação adaptada às características dos estudantes em um ambiente inteligente de aprendizagem. 
VII Congresso Brasileiro de Informática na Educação (CBIE 2018)

Anais do XXIX Simpósio Brasileiro de Informática na Educação (SBIE 2018)

\section{Método}

O objetivo geral deste trabalho é verificar e validar a hipótese de que a utilização de técnicas de mindfulness, adaptadas ao humor e ao tempo de prática do estudante em um STI, pode melhorar a atenção do estudante e resultar em uma melhora na aprendizagem. Para alcançar esse objetivo, este trabalho implementou um agente computacional, integrado ao STI PAT2Math, que objetiva fornecer ao estudante um treinamento guiado e adaptado de mindfulness, a fim de melhorar sua atenção. De forma geral, o agente desenvolvido apresenta ao aluno gravações de áudio que fornecem uma forma de meditação guiada, visando melhorar a atenção do aluno e levar à reflexão de como está a sua atenção e quais ações o próprio estudante pode tomar para melhorar a habilidade de focar sua atenção nas atividades escolares.

Esta seção traz informações sobre os participantes, os materiais, o desenho experimental e o procedimento empregados no experimento.

\subsection{Participantes}

Participaram do experimento 41 estudantes $(M=22$ e $F=19)$ do sétimo ano de um colégio particular no estado do Rio Grande do Sul, oriundos de três turmas distintas. A idade dos participantes oscilou entre 12 e 14 anos, com média de 13 anos. O conteúdo matemático presente no STI utilizado esteve em consonância com as diretrizes de ensino da escola e foram trabalhados pela professora de Matemática durante o estudo. A professora de Matemática era a mesma para as três turmas. Os dados de alunos que não participaram do pré e pós-teste e de pelo menos quatro sessões no STI foram desconsiderados. Dessa forma, dos 64 alunos que iniciaram o experimento, foram considerados os dados de apenas 41 alunos.

\subsection{Materiais}

Os materiais utilizados durante o experimento foram: (1) questionário de atenção, apresentado aos alunos em todas as sessões para os três grupos no início da sessão, no meio e ao final; (2) questionário de estado de humor, apresentado aos alunos do grupo experimental no início da sessão; (3) um questionário de avaliação do agente, apresentado aos alunos dos três grupos apenas ao final do experimento; (4) pré e pós-teste, respondidos por todos os alunos no primeiro e no último encontro do experimento, respectivamente; e, por fim, (5) as gravações de áudio, que eram escutadas pelos alunos durante as sessões de utilização do STI, onde cada grupo escutava seu respectivo tipo de áudio.

1. Questionário de Atenção - Um psicólogo com experiência pregressa em psicometria e mindfulness desenvolveu seis itens relacionados à atenção para que os alunos pudessem pontuá-los de 1 a 10, seguindo uma escala Likert, de acordo com suas percepções acerca do seu estado de atenção no momento que eram questionados. O questionário é baseado em instrumentos utilizados para avaliar o nível de atenção em programas de mindfulness cujo o público alvo são crianças e adolescentes em idade escolar. Destes instrumentos, a principal referência é o MAAS-A (Mindful Attention Awareness Scale for Adolescents) [Brown et al. 2011]. Objetivou-se, assim, mensurar traços comportamentais do construto psicológico de atenção. Em cada sessão eram selecionados três itens que eram apresentados ao aluno. Um exemplo de item é "Neste momento estou focado para realizar as atividades". 
VII Congresso Brasileiro de Informática na Educação (CBIE 2018)

Anais do XXIX Simpósio Brasileiro de Informática na Educação (SBIE 2018)

2. Questionário de Estado de Humor - Esse questionário era apresentado ao aluno com o objetivo de identificar o seu estado de humor por meio de autorrelato. $\mathrm{O}$ questionário é composto por uma única questão de múltipla escolha, restrito aos estados: feliz, distraído, entediado e bravo. Para facilitar a seleção do estado de humor, foram utilizadas imagens, em forma de emojis, para que os alunos selecionassem a imagem que melhor representasse seu estado atual.

3. Questionário de Avaliação do Agente - Para a avaliação do agente foi elaborado um questionário com nove questões, por exemplo, "Os áudios deixaram as atividades dentro do PAT2Math mais interessantes". Esse foi respondido pelos alunos na sessão que antecedeu a realização do pós-teste. Esses itens buscaram avaliar o quanto a versão do STI integrada ao agente, que utilizava as técnicas de meditação guiadas e adaptadas, era mais efetiva em relação às outras duas versões utilizadas pelos grupos de controle. As respostas das questões seguiam uma escala Likert, variando entre 1 (discordo plenamente) e 5 (concordo plenamente). Por não ter sido encontrado um documento em português devidamente validado para a elaboração deste instrumento foi optado por desenvolver um instrumento próprio.

4. Pré e pós-teste: Os testes tinham como objetivo verificar e validar a hipótese de que a prática de meditação guiada e adaptada pode produzir um efeito positivo na aprendizagem, mesmo de forma indireta. Estes testes, valendo 10, foram realizados em forma de uma prova em papel e caneta composta por 15 equações algébricas de primeiro grau que deveriam ser respondidas pelos alunos participantes de forma individual e sem consulta. Os testes foram aplicados a todos os alunos, independente de seu grupo. A complexidade das questões era equivalente para ambos os testes, bem como a forma de correção dos mesmos.

5. Áudios: Todos os áudios foram gravados pelo mesmo narrador (um profissional com experiência prévia em radialismo) e foram classificadas em três categorias:

(a) História da álgebra: Estes áudios tinham duração média de 5 minutos e contavam alguma história relacionada à Álgebra;

(b) Meditação não-adaptada: Estes áudios reproduziam técnicas de mindfulness guiadas, com uma duração de aproximadamente 5 minutos, porém a seleção dos áudios era realizada de forma aleatória, sem nenhum tipo de adaptação;

(c) Meditação adaptada: Os áudios de meditação adaptados eram de três, quatro ou cinco minutos de duração. Haviam também áudios com diferentes técnicas de meditação mindfulness guiadas, tais como técnicas de atenção plena à respiração, técnicas de atenção aos sentidos (visão, audição, tato e olfato) e técnicas de escaneamento corporal. Tais técnicas são comumente utilizadas em programas de redução de estresse baseados em mindfulness e que têm fortes evidências científicas de sua eficiência [Shapiro et al. 2008].

\subsection{Adaptação do Agente}

A parte da adaptação foi implementada somente no agente descrito nesse artigo. Apenas o grupo experimental utilizou esse agente, que era responsável pela seleção dos áudios que seriam apresentados aos alunos baseado em seu humor e seu tempo de experiência 
VII Congresso Brasileiro de Informática na Educação (CBIE 2018)

Anais do XXIX Simpósio Brasileiro de Informática na Educação (SBIE 2018)

em meditação. Sobre a adaptação do humor, inicialmente, o aluno respondia ao questionário de estado de humor, conforme descrito na sessão de materiais. Para cada tipo de humor, havia um grupo de áudios de meditação que o agente poderia selecionar para apresentar ao aluno. Por exemplo, respiração atenta e escaneamento corporal eram apresentados quando o aluno estivesse distraído, já a meditação da paz ou previsão do tempo interior eram introduzidos quando ele estivesse bravo ou chateado, etc. Sobre a adaptação do tempo, quanto mais tempo de experiência o aluno possuía, maior eram os áudios selecionados para esse aluno. A adaptação progressiva da duração das meditações se mostra uma alternativa interessante, pois apesar das práticas de mindfulness promoverem diversos benefícios aos estudantes, permanecer sentados em uma mesma posição ou focar em um único estímulo pode ser difícil para os estudantes, gerando frustração e se mostrando inadequado ao experimento [Lillard 2011].

\subsection{Desenho Experimental}

Foi realizada uma avaliação para verificar a validade da hipótese deste trabalho. Essa avaliação seguiu um desenho experimental, formado por dois grupos de controle e um grupo experimental, totalizando três grupos, aos quais os alunos eram atribuídos de forma aleatória. O primeiro grupo de controle, controle 1, formado por 12 alunos, ouviu apenas áudios que narravam a história da Álgebra. O segundo grupo de controle, controle 2, formado por 11 alunos, ouviu áudios de técnicas de meditação não adaptadas, sendo estas selecionadas aleatoriamente. Por fim, o grupo experimental, formado por 18 alunos, utilizou o agente desenvolvido neste trabalho, que conta com a utilização de técnicas guiadas e adaptadas ao humor e ao tempo de prática do estudante.

A escolha desses três grupos permitiu avaliar duas situações distintas:

1. Grupo Controle 1 (História da álgebra) versus Grupo Experimental - Esta comparação permite verificar se a prática de meditação guiada e adaptada produz efeitos positivos na capacidade de atenção do estudante quando comparada ao uso de outros áudios com finalidade educacional ou pedagógica.

2. Grupo Controle 2 (Meditação não adaptada) versus Grupo Experimental Este cenário permite verificar se a prática de meditação guiada e adaptada ao humor e à experiência do aluno pode produzir efeitos mais positivos na atenção do aluno, quando comparada com a utilização de técnicas de meditação não adaptadas.

\subsection{Procedimento}

A avaliação ocorreu durante 7 semanas, onde em cada semana foi realizada uma sessão com duração de 50 minutos. Na primeira e na última sessão os estudantes realizaram o pré e pós-teste, respectivamente. Nas sessões de 2 a 6 , os alunos seguiram o seguinte roteiro: ao se logar no sistema, os alunos respondiam o questionário de atenção (denominado QA_V1) e, somente para o grupo experimental, o questionário de estado de humor. Após, o aluno deveria escutar um áudio (de 3-5 minutos), dependendo do grupo em que se encontrava (poderia ser um conto sobre história da álgebra (controle 1), uma meditação mindfulness (controle 2) ou uma técnica adaptada de meditação que dependia do humor relatado pelo aluno (experimental)). Após escutar a gravação de áudio (o aluno não conseguia ir para a próxima etapa, antes que a reprodução do áudio se encerrasse), o estudante deveria responder o questionário de atenção novamente (denominado QA_V2). 
VII Congresso Brasileiro de Informática na Educação (CBIE 2018)

Anais do XXIX Simpósio Brasileiro de Informática na Educação (SBIE 2018)

Em seguida, o aluno era direcionado para a interface do STI PAT2Math, onde iniciava a resolução de equações algébricas. Ao final da sessão no STI, o questionário de atenção era apresentado novamente ao estudante (denominado QA_V3). Todos as respostas dos questionários respondidos pelos alunos eram armazenadas no modelo do aluno. A Figura 1 ilustra as etapas ocorridas durante o experimento.

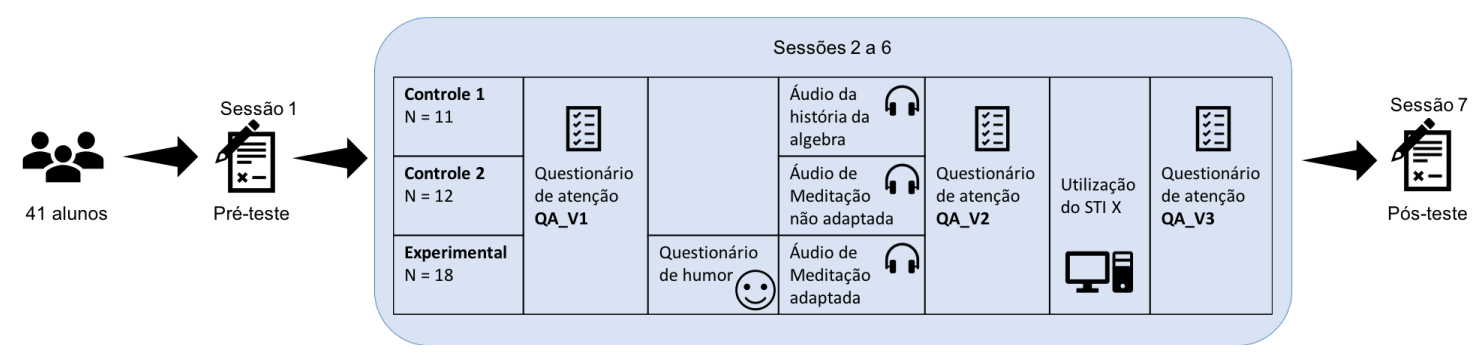

Figura 1. Etapas do experimento

\section{Resultados e Discussão}

Esta seção descreve os resultados obtidos no experimento realizado, conforme descrito na seção anterior. Os resultados vêm de três materiais em específico: (i) pré e pós-teste, $(i i)$ questionários de atenção e (iii) questionário de avaliação do agente.

Com o pré e pós-teste, o objetivo era identificar se a prática de meditação guiada e adaptada pode produzir um ganho maior de aprendizagem que a meditação não adaptada ou ouvir um áudio (história de álgebra). Ao analisar a diferença dos resultados entre pré-teste do grupo de controle $1(M=5,06$ e $D P=2,61)$, controle $2(M=5,99$ e $D P=1,57)$ e experimental $(M=6,02$ e $D P=1,60)$ com os resultados do pósteste do grupo de controle $1(M=4,99$ e $D P=2,74)$, controle $2(M=5,91 \mathrm{e}$ $D P=1,49)$ e experimental $(M=5,96$ e $D P=1,34)$, verifica-se que não há ganho de aprendizagem entre os grupos e, por isso, nenhuma diferença significativa foi encontrada. Acredita-se que a curta duração do experimento, bem como a proximidade do final do ano letivo e a falta de alunos que já estavam aprovados, afetou os resultados. Assim sendo, não há evidências de um ganho maior de aprendizagem para o grupo que teve meditação adaptada.

Nos questionários de atenção, o objetivo era identificar se a prática de mindfulness guiada e adaptada poderia afetar a atenção dos alunos. Para isso, esse questionário era apresentado ao aluno no início da sessão (QA_V1), após ouvir o áudio de meditação (QA_V2) e após a utilização do STI PAT2Math (QA_V3). As questões são relacionadas à atenção como um todo, porém algumas possuem valência inversa, o que foi contabilizado antes do cálculo dos resultados. A Figura 2 apresenta a média dos resultados obtidos em cada um dos questionários no decorrer das 5 sessões de utilização do STI.

É possível verificar nos gráficos da Figura 2, que a média dos resultados do QA_V2 e QA_V3 são mais altos do que o QA_V1, para ambos os grupos, apontando que qualquer intervenção por áudio trouxe uma melhora na atenção dos alunos. Porém, nota-se que o grupo experimental obteve uma média maior, mais duradoura e mais constante em relação aos grupos de controle. É interessante ressaltar que, a partir do segundo dia de experimento, o grupo experimental foi superior ao grupo de controle com meditação não 
VII Congresso Brasileiro de Informática na Educação (CBIE 2018)

Anais do XXIX Simpósio Brasileiro de Informática na Educação (SBIE 2018)

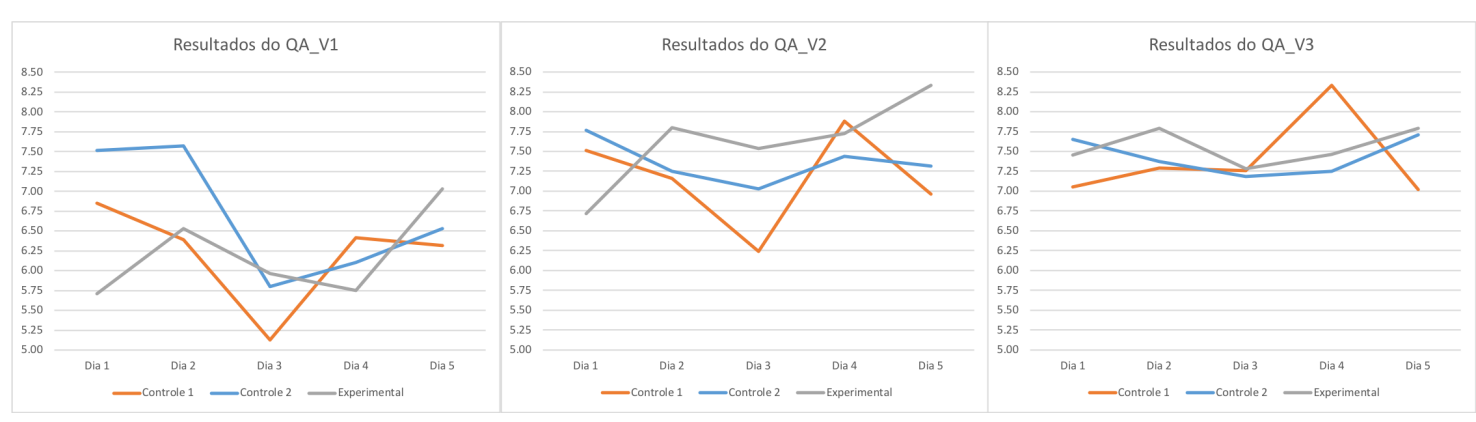

Figura 2. Resultados das três aplicações do questionário de atenção

adaptada. O grupo de controle com história da álgebra apresenta um comportamento imprevisível, indicando que, embora afete a atenção dos alunos, não traz uma constância para a mesma.

Na última sessão de utilização do STI, antes do pós-teste, o questionário de avaliação do agente foi aplicado aos alunos. Primeiramente, foram analisados os dados dos três grupos separadamente. Como não foi encontrada uma diferença estatisticamente significativa para essa comparação, os dados dos dois grupos de controle foram agrupados em um único e comparados com os dados do grupo experimental. Esses dados foram verificados com o teste de normalidade Shapiro Wilk, apresentando distribuições não normais. Dessa forma, foi empregado o teste não-paramétrico Wilcoxon Runk Sum (versão não paramétrica do teste $t$ independente) para análise estatística. Igualmente, foi calculado o tamanho do efeito para os resultados das comparações. Segundo [Field et al. 2012], "um tamanho de efeito é simplesmente uma medida objetiva e (geralmente) padronizada da magnitude do efeito observado". As medidas de efeito mais utilizadas são o $d$ de Cohen e o coeficiente de correlação de Pearson $r$. Este trabalho utiliza o coeficiente de Pearson para a análise de efeito, conforme sugerido por [Field et al. 2012]. Os valores utilizados são: $r=0,10$ representa um efeito pequeno, $r=0,30$ representa um efeito médio e $r=0,50$ representa um efeito grande.

Por meio da análise dos resultados obtidos nesse questionário, foi possível identificar quatro aspectos que obtiveram relevância estatística sobre a aplicação de técnicas guiadas e adaptadas de mindfulness em um STI (ver Tabela 1). (i) Quando questionados se os alunos gostaram de utilizar o PAT2Math com áudio, o grupo experimental apresentou um resultado superior aos dois grupos de controle. Comparando os resultados obtidos dos dois grupos de controle com o grupo experimental, foi encontrado um valor de $p=0,0247$, com $W=133,5$ e $r=-0,3506$, indicando uma diferença estatisticamente significativa. (ii) Os alunos também relataram sobre a utilidade dos áudios no STI. Novamente, foi realizada uma comparação entre os dois grupos de controle com o grupo experimental. O grupo experimental considerou os áudios no STI mais úteis ( $W=136$, $p=0,0278$ e $r=-0,3436$ ). (iii) Os estudantes também foram questionados se o uso dos áudios tornou mais fácil o uso do STI. Os alunos do grupo experimental estavam mais de acordo com a afirmação de que os áudios (as meditações guiadas e adaptadas) facilitaram o uso do STI que os alunos dos grupos de controle. Por fim, é possível afirmar, com segurança estatística, que (iv) os alunos que praticaram as técnicas de mindfulness guiadas e adaptadas se sentiram mais interessados nos estudos $(p=0,0205$, com $W=130$ e $r=-0,3617)$. Esses resultados trazem evidências que os estudantes consideram as 
VII Congresso Brasileiro de Informática na Educação (CBIE 2018)

Anais do XXIX Simpósio Brasileiro de Informática na Educação (SBIE 2018)

técnicas de meditação guiadas e adaptadas um recurso útil, que aumentou o interesse deles no estudo e no STI.

Tabela 1. Resultados significativos obtidos do questionário final

\begin{tabular}{|c|c|c|c|c|c|c|c|}
\hline $\mathbf{Q}$ & Pergunta & Respostas & Grupo & M & DP & Wilcoxon & Efeito \\
\hline \multirow{2}{*}{1} & \multirow{2}{*}{$\begin{array}{l}\text { Você gostou de utilizar a versão } \\
\text { do PAT2Math com áudio? }\end{array}$} & \multirow{2}{*}{$\begin{array}{l}\text { gostei/ } \\
\text { não gostei }\end{array}$} & Controles 1 e 2 & 3,75 & 0,99 & \multirow{2}{*}{$\begin{array}{l}\mathrm{p}=0,0247 \\
\mathrm{~W}=133,5\end{array}$} & \multirow{2}{*}{506} \\
\hline & & & Experimental & 4,24 & 1,20 & & \\
\hline \multirow{2}{*}{2} & \multirow{2}{*}{ Você acredita que os áudios são: } & úteis/ & Controles 1 e 2 & 3,21 & 1,28 & \multirow{2}{*}{$\begin{array}{c}\mathrm{p}=0,0278 \\
\mathrm{~W}=136\end{array}$} & \multirow{2}{*}{$r=-0,3436$} \\
\hline & & não úteis & Experimental & 3,94 & 1,44 & & \\
\hline \multirow{2}{*}{3} & \multirow{2}{*}{$\begin{array}{l}\text { O uso dos áudios facilitaram o } \\
\text { uso do PAT2Math: }\end{array}$} & \multirow{2}{*}{$\begin{array}{l}\text { concordo/ } \\
\text { discordo }\end{array}$} & Controles 1 e 2 & 3,37 & 1,06 & \multirow{2}{*}{$\begin{array}{c}\mathrm{p}=0,0132 \\
\mathrm{~W}=123\end{array}$} & \multirow{2}{*}{$r=-0,387$} \\
\hline & & & ental & 4,11 & 0,93 & & \\
\hline \multirow{2}{*}{4} & \multirow{2}{*}{$\begin{array}{l}\text { Os áudios aumentaram o meu } \\
\text { interesse nos estudos: }\end{array}$} & \multirow{2}{*}{$\begin{array}{l}\text { concordo/ } \\
\text { discordo }\end{array}$} & Controles 1 e 2 & 3,13 & 1,03 & \multirow{2}{*}{$\begin{array}{c}\mathrm{p}=0,0205 \\
\mathrm{~W}=130\end{array}$} & \multirow{2}{*}{$r=-0,3617$} \\
\hline & & & Experimental & 3,82 & 1,13 & & \\
\hline
\end{tabular}

Além das análises quantitativas, foram realizadas entrevistas com cinco alunos ( $\mathrm{F}$ $=4$ e $\mathrm{M}=1$ ) do grupo experimental. Os alunos foram indicados pela professora responsável por possuírem diferentes níveis de proficiência em Álgebra (desempenho fraco, médio e excelente) e concordaram em relatar sua experiência de utilizar as técnicas de mindfulness em um STI. A entrevista foi conduzida por um formando do curso de psicologia e validada por um profissional da área da psicologia. Os relatos foram similares aos resultados encontrados no questionário de avaliação do agente. Todos os entrevistados relataram se sentir mais atentos e concentrados, além de perceber a mudança da postura de alguns colegas considerados mais agitados na turma.

\section{Conclusão}

O agente computacional proposto nesse trabalho visa, através de técnicas de meditação guiadas e adaptadas, melhorar a atenção e, consequentemente, a aprendizagem durante a resolução de equações algébricas em um sistema tutor inteligente. Além disso, ele objetiva igualmente despertar no aluno uma consciência de que sempre que ele se perceber distraído ele tem ao seu alcance vários elementos para tornar a direcionar sua atenção e consciência para o momento e para a tarefa atual.

Uma avaliação experimental realizada com 41 estudantes do $7^{\circ}$ ano do ensino fundamental encontrou evidências de que o uso de técnicas de meditação guiadas e adaptadas podem melhorar a experiência de utilização dos sistemas tutores inteligentes. Além disso, em relação à melhora dos níveis de atenção, a análise dos dados colhidos durante as 5 semanas de avaliação do agente mostra que a utilização de técnicas de meditação guiada e adaptada podem produzir uma melhora na capacidade de atenção dos estudantes.

Como trabalhos futuros pretende-se estudar as possibilidades de melhoria no sistema de identificação de humor e de outros fatores que podem fornecer dados mais precisos sobre o comportamento do aluno antes, durante e após a prática de meditação. $\mathrm{O}$ agente atual utiliza um questionário de autorrelato para identificar o humor do aluno. A adição de um sistema capaz de reconhecer e analisar padrões nas expressões faciais e na movimentação do estudante quando sentado na cadeira podem ser formas menos intrusivas e mais efetivas de deteç̧ão. Busca-se também adicionar um agente pedagógico animado, que seja capaz de dar suporte ao estudante sem ser excessivamente intrusivo, para aumentar o engajamento e a motivação dos estudantes para praticar técnicas de meditação dentro e fora de um sistema tutor inteligente. 
VII Congresso Brasileiro de Informática na Educação (CBIE 2018)

Anais do XXIX Simpósio Brasileiro de Informática na Educação (SBIE 2018)

\section{Referências}

Bostic, J. Q. (2015). Being Present at School. Implementing Mindfulness in Schools. Child and Adolescent Psychiatric Clinics of North America, 24(2):245-259.

Brown, K. W., West, A. M., Loverich, T. M., and Biegel, G. M. (2011). Assessing adolescent mindfulness: Validation of an adapted mindful attention awareness scale in adolescent normative and psychiatric populations. Psychological assessment, 23(4):1023.

D’Mello, S. (2016). Attending to Attention : Detecting and Combating Mind Wandering during Computerized Reading. CHI Extended Abstracts on Human Factors in Computing Systems, pages 1661-1669.

Field, A., Miles, J., and Field, Z. (2012). Discovering statistics using R. Sage.

Goldsby, T. L., Goldsby, M. E., McWalters, M., and Mills, P. J. (2017). Effects of singing bowl sound meditation on mood, tension, and well-being: An observational study. Journal of Evidence-based Complementary \& Alternative Medicine, 22(3):401-406.

Gromala, D., Tong, X., Choo, A., Karamnejad, M., and Shaw, C. D. (2015). The Virtual Meditative Walk: Virtual Reality Therapy for Chronic Pain Management. ACM Conference on Human Factors in Computing Systems, pages 521-524.

Hudlicka, E. (2013). Virtual training and coaching of health behavior: Example from mindfulness meditation training. Patient Education and Counseling, 92(2):160-166.

Jaques, P. A., Seffrin, H., Rubi, G., de Morais, F., Ghilardi, C., Bittencourt, I. I., and Isotani, S. (2013). Rule-based expert systems to support step-by-step guidance in algebraic problem solving. Expert Systems with Applications, 40(14):5456-5465.

Kabat-Zinn, J. (2003). Mindfulness-based interventions in context: past, present, and future. Clinical psychology: Science and practice, 10(2):144-156.

Ladewig, I. (2017). A importância da atenção na aprendizagem de habilidades motoras. Revista Paulista de Educação Física, (supl. 3):62-71.

Lillard, A. S. (2011). Mindfulness practices in education: Montessori's approach. Mindfulness, 2(2):78-85.

Schaab, B. L., Duarte, M., Azevedo, O. B., Abs, D. V., and Jaques, P. A. (2015). Aplicação do mindfulness em um sistema tutor inteligente: um estudo piloto. In $C B I E$ - SBIE), number 1 .

Shapiro, S. L., Oman, D., Thoresen, C. E., Plante, T. G., and Flinders, T. (2008). Cultivating mindfulness: effects on well-being. Journal of clinical psychology, 64(7):840862.

Tram, J. S. (2017). The effects of mindfulness on the on-task behavior of students with disabilities. $\mathrm{PhD}$ thesis, California State University.

Waldemar, J. O. C., Rigatti, R., Menezes, C. B., Guimarães, G., Falceto, O., and Heldt, E. (2016). Impact of a combined mindfulness and social-emotional learning program on fifth graders in a brazilian public school setting. Psychology \& Neuroscience, 9(1):79.

Yuzheng, W., Wei, X., Capella, Z., and Xinghua, L. (2017). Does mind wandering mediate the association between mindfulness and negative mood? a preliminary study. Psychological Reports, 120(1):118 - 129. 